\title{
Measurement of Nonlinearity and Spectral Study of a Laser Dye
}

\section{Sindhu Sukumaran $\mathbf{V}^{1 *}$, Ramalingam $\mathbf{A}^{2}$ and Srinivasan $\mathbf{A G}^{3}$}

${ }^{1}$ Central Institute of Plastics Engineering and Technology, Guindy, Chennai

${ }^{2}$ Department of Physics Centre for Laser Technology, Anna University, Chennai-600 025 India

${ }^{3}$ Dr.M.G.R.Educational and Research Institute, Maduravoil Chennai

\begin{abstract}
Problem statement: The third order nonlinear optical properties of organic Polymers are used in high speed telecommunications, optical limiters and optical; computes. The purpose of study was to determine the spectral and third order nonlinear characteristics of the organic dye, Victoria blue in liquid and in solid medium.

Approach: The samples were prepared by dissolving the dye in n-butyl acetate (liquid medium) and a thin film of thickness $1 \mathrm{~mm}$ was prepared by bulk polymerization method. The spectral characteristics of victoria blue dye doped poly (methyl methacrylate) modified with additive n-butyl acetate (nBA) are studied by recording its absorption and fluorescence spectra and the results are compared with the corresponding liquid mixture. The nonlinear refractive index of the dye in nBA and dye doped polymer film were measured using z-scan technique [1], by exciting with HeNe laser. The results obtained are intercompared.

Results: Both the samples of dye Victoria blue in liquid and solid medium show a negative nonlinear refractive index. A negative nonlinear dye doped films can be used as optical limiters. The origin of optical nonlinearity in the dye may be attributed due to laser-heating induced nonlinear effect.
\end{abstract}

PACS Code: 42.55.Mv, 42.65.-k, 82.35.Ej, 42.70.Jk

Keywords: Victoria blue : Solid dye laser; nBA; MMA; Victoris bluet; Nonlinear refractive index

\section{Introduction}

Rapid technological developments in optics have placed great demand on the development of nonlinear optical (NLO) materials [1]. The use of solid state dye lasers gets rid of many of the common problems associated with static or flowing liquid systems. The most frequently used polymeric material is polymethylmethacrylate (PMMA) [2]. The low solubility of dye in PMMA causes limitations, which is overcome by introducing modifying additives. A review of literature showed that most of the work on dye-doped polymers has been done with Rhodamine dyes [3] and pyromethane dyes. Some works are reported on coumarin dyes [2]. The study of nonlinear refractive index on dye IR140 [4], has been reported. No work has been reported on the study of nonlinear refractive index of the dye Victoria blue. The physical origin of nonlinear refraction can be electronic, molecular, electrostrictive or thermal. Historically, the first optical limiter was based on thermal mechanism with a cw laser [5]. Recently, thermal effects have been shown to be efficient even with nanosecond pulses [6,7] In this paper, we have studied the spectral characteristics and nonlinear properties of the dye Victoria blue in both the polymer and the corresponding monomer compositions in detail.

\section{Experimental Procedure}

The laser dye, Victoria blue obtained from Exciton Inc., USA was chosen for this study. Thin layer chromatography (TLC) test confirms the absence of any impurities in this dye. Methyl methacrylate (MMA) (Lancaster) was used as monomer. Initial MMA compositions were cleared of foreign inclusions. Spectroscopic grade n-butyl acetate (nBA) purchased from Merck (India) was chosen as an additive because it combines good solubility for victoria blue dye.

\section{Synthesis of dye-doped polymer rod and film}

The dye-doped polymer rod of dye concentration $0.05 \times 10^{-3} \mathrm{M}$ was synthesized by thermal bulk free radical polymerization method [8].
MMA and nBA were taken in the ratio 4:1(v/v). Known weight of dye was dissolved in this mixture. $3 \mathrm{~g}$ of 2,2-azobis (isobutyronitrile) per litre of MMA solution was used as an initiator for polymerization. The solution was put into a polymerization tube and kept in the nitrogen atmosphere. Bulk polymerization was carried out in a temperature controlled water bath and the temperature was maintained at $35^{\circ} \mathrm{C}$ for 2 days, at $40^{\circ} \mathrm{C}$ for another 2 days and at $50^{\circ} \mathrm{C}$ for 7 days. The polymerized rod was removed by breaking the glass tube. Rod (length $3 \mathrm{~cm}$ : diameter $1 \mathrm{~cm}$ ) obtained, was cut, ground and polished to optical quality required. The internal optical qualities of polymer rod were checked by passing the He-Ne laser beam of $5 \mathrm{~mW}$ through the rod. No dispersion or distortion of the He-Ne laser beam was observed. The dye doped polymer thin film of concentration $0.05 \times 10^{-3} \mathrm{M}$ was prepared by pouring the viscous dye solution with initiator mixture on to a glass slide placed inside a glass enclosure, kept at a temperature of $40^{\circ} \mathrm{C}$ in temperature controlled water bath.

\section{Spectral characteristics, quantum yield}

The spectral properties of the dye are studied by recording the absorption and fluorescence spectra of dye in MMA, in $\mathrm{nBA}$, in a mixture of MMA and nBA (liquid medium) and in the solid matrix (PMMA modified with nBA) using Hitachi U2000 spectrophotometer and Hitachi F2000 spectrofluorometer respectively. The fluorescence spectra were corrected using quinine sulphate in $0.1 \mathrm{~N}_{2} \mathrm{SO}_{4}$ and fluorescein in $0.1 \mathrm{~N} \mathrm{NaOH}$ [9]. Care was taken to record all spectra

${ }^{*}$ Corresponding author: Sindhu Sukumaran V, Department of Physics, Faculty, CIPET, Chennai, Tel: 091-44- 2638 0438; E-mail: sindhusukumaran@yahoo.co.in

Received June 25, 2015; Accepted July 27, 2015; Published July 29, 2015

Citation: Sindhu SV, Ramalingam A, Srinivasan AG (2015) Measurement of Nonlinearity and Spectral Study of a Laser Dye. J Laser Opt Photonics 2: 121. doi:10.4172/2469-410X.1000121

Copyright: (c) 2015 Sindhu SV. This is an open-access article distributed under the terms of the Creative Commons Attribution License, which permits unrestricted use, distribution, and reproduction in any medium, provided the original author and source are credited. 
under identical conditions. These spectra are shown in Figures 1 and 2. Quantum yields [10] were calculated using Rhodamine 6G $(\mathrm{Rh}$ $6 \mathrm{G}$ ) in ethanol as the fluorescence standard with refractive index and differential absorption correction. The absorbance of Rh $6 \mathrm{G}$ in ethanol at $500 \mathrm{~nm}$ is 0.66 and the reported quantum yield of the dye Rh $6 \mathrm{G}$ is 0.95 [11]. The spectral parameters such as absorption peak wavelength (peak $\lambda)$, molar extinction coefficient $(€)$, bandwidth $\left(\Delta \ddot{y}_{1 / 2}\right)$, oscillator strength (F),fluorescence peak wavelength (peak $\lambda)$, full width at half maximum (FWHM), fluorescence quantum yield $\left(\varnothing_{\mathrm{f}}\right)$, Stoke's shift of the dye were calculated and are shown in Table 1.

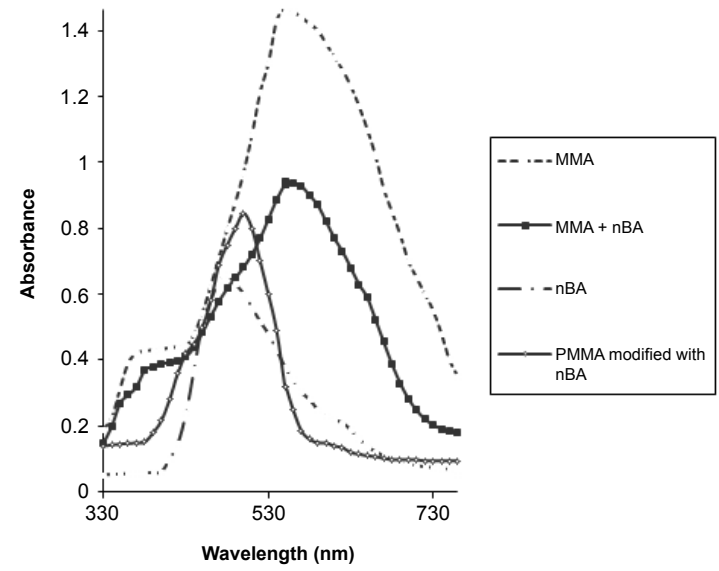

Figure 1: Absorption spectra of dye Victoria blue in (a) MMA (b) MMA+nBA (c) nBA (d) $P M M A+n B A$.

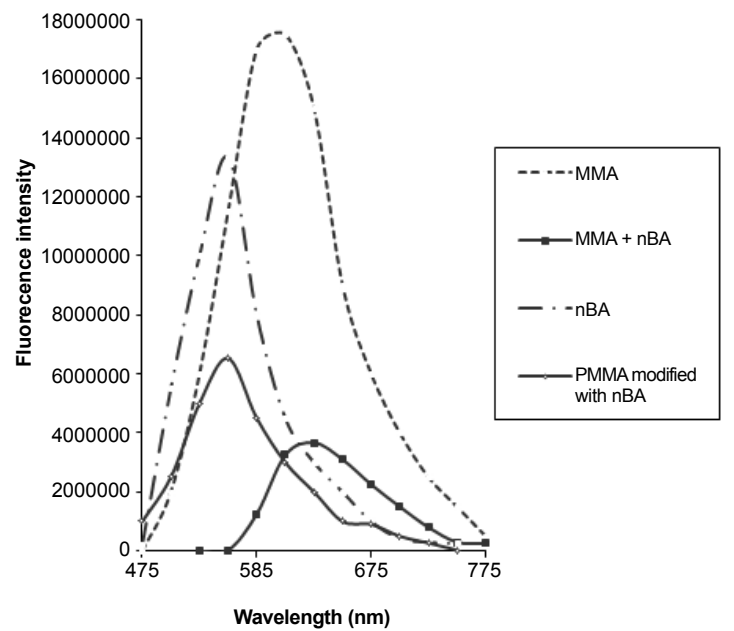

Figure 2: Fluorescence spectra of dye Victoria blue in (a) MMA (b) MMA+nBA (c) nBA (d) PMMA+nBA

\section{Nonlinear studies}

The closed z-scan set up is shown in Figure 3. A He-Ne laser $(632.8 \mathrm{~nm}$, power $10 \mathrm{~mW})$ was used as the light source. The dye doped polymer film was used as sample of thickness $1 \mathrm{~mm}$. The transmittance of the nonlinear medium through a finite aperture in the far field as a function of the sample position $\mathrm{z}$ was measured by using a positive lens having a focal length $\mathrm{f}=4 \mathrm{~cm}$. The results obtained are compared with the dye in $\mathrm{nBA}$. The liquid was filled in the sample cell with a path length of $1 \mathrm{~mm}$. The beam waist $\left(\omega_{0}\right)$ of He-Ne laser was calculated using the formula $\omega_{0}=1.22 \mathrm{~d} / \mathrm{d}(\mathrm{d}$ is the diameter of the aperture in front of the focal lens, $f$ is the focal length of the convex lens used in the set up) and is found to be $75 \mu \mathrm{m}$ with a Rayleigh range of $27.91 \mathrm{~mm}$. The transmitted energy was measured using PMT (RCA 931) connected to an oscilloscope (OS768A) in a far field. Care was taken that PMT does not get saturated.

As the monomer (MMA) does not introduce any nonlinearity, the experiment was performed only for the dye in nBA. Figures 4 and 5 show the $\mathrm{z}$-scan of the dye victoria blue in $\mathrm{nBA}$ and thin polymer film respectively. Figure 6 shows the optical limiting curves obtained with a He-Ne laser input of wavelength $632.8 \mathrm{~nm}$ for victoria blue in solvent nBA. The samples show very good optical limiting behavior arising from nonlinear refraction. The output power rises initially with increase in input power, but after a certain threshold value the samples start defocusing the beam resulting in a greater part of the beam crosssection being cut off by the aperture. Thus the transmittance recorded by the photo detector remained reasonably constant showing a plateau region.

\section{Results}

The absorption and fluorescence spectral profiles of the dye in liquid and in solid media are identical. This indicates that at low concentrations, the dye exists only in its monomeric form inside a polymer matrix and that at such low concentration there is a given amount of free volume within the polymeric matrix in which the dye molecules distribute in a way to what happens in a diluted liquid solution of the dye [12].

The peak wavelength of absorption and fluorescence of victoria blue in the solid matrix shows a blue shift from that in the liquid media. These changes may be attributed to the structure of dye. The spectral changes of the dye in the solid matrix and liquid media may also be due to the changes in the dielectric constant of the respective media.

The absorption bandwidth and the fluorescence bandwidth of the dye victoria blue are broader in solid matrix than in liquid medium. The spectral characteristics of the lasing dye in polymer depend on the intermolecular interaction between the dye molecule and macromolecules.

The Stoke's shift of the dye in solid matrix is more than that in the liquid medium. The Stoke's shift of the dye molecule in the solid matrix depends on the difference in the dipole moments between the

\begin{tabular}{|c|c|c|c|c|c|c|c|}
\hline \multirow[t]{2}{*}{ Solvent/Medium } & \multicolumn{3}{|c|}{ Absorption spectra } & \multirow[b]{2}{*}{$\begin{array}{l}\text { Oscillator Strength } \mathrm{f} \\
10^{-24} \mathrm{~L} \mathrm{~mol}^{-1} \mathrm{~cm}^{-2}\end{array}$} & \multicolumn{3}{|c|}{ Fluorescence spectra } \\
\hline & $\begin{array}{l}\text { Peak wavelength } \\
\text { in } \mathrm{nm}\end{array}$ & $\frac{\varepsilon}{10^{4} \mathrm{~L} \mathrm{~mol}^{-1} \mathrm{~cm}^{-1}}$ & $(\Delta v)_{1 / 2} \mathrm{~cm}^{-1}$ & & $\begin{array}{l}\text { Peak wavelength } \\
\text { in } \mathrm{nm}\end{array}$ & FWHM nm & Stoke's shift $\mathrm{cm}^{-1}$ \\
\hline MMA & 550 & 2.934 & 4430.3 & 0.5628 & 585 & 96.25 & 1087.8 \\
\hline MMA and nBA & 560 & 1.908 & 5615.2 & 0.4639 & 628 & 105 & 1933.5 \\
\hline nBA & 480 & 1.318 & 5267.9 & 0.3 & 555 & 116.25 & 2815.3 \\
\hline PMMA modified with nBA & 500 & 1.6386 & 5429.8 & 0.397 & 552 & 115 & 1884.05 \\
\hline
\end{tabular}

Table 1: Spectral characteristics, quantum yield, of the dye Victoria. 


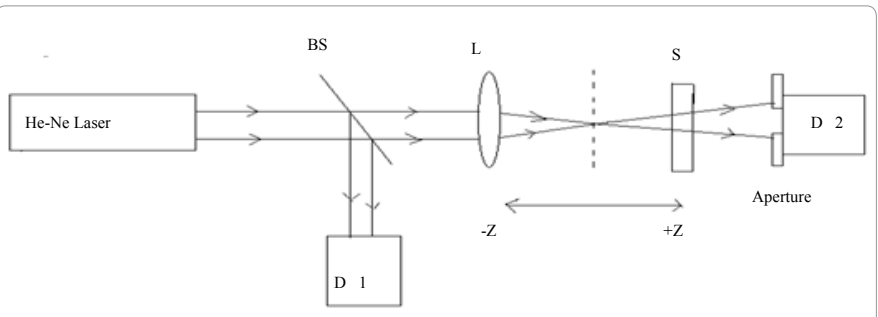

Figure 3: The closed z-scan set up. D1, D2: Detectors; BS: Beam Splitter; L: Convex Lens; S: Sample.

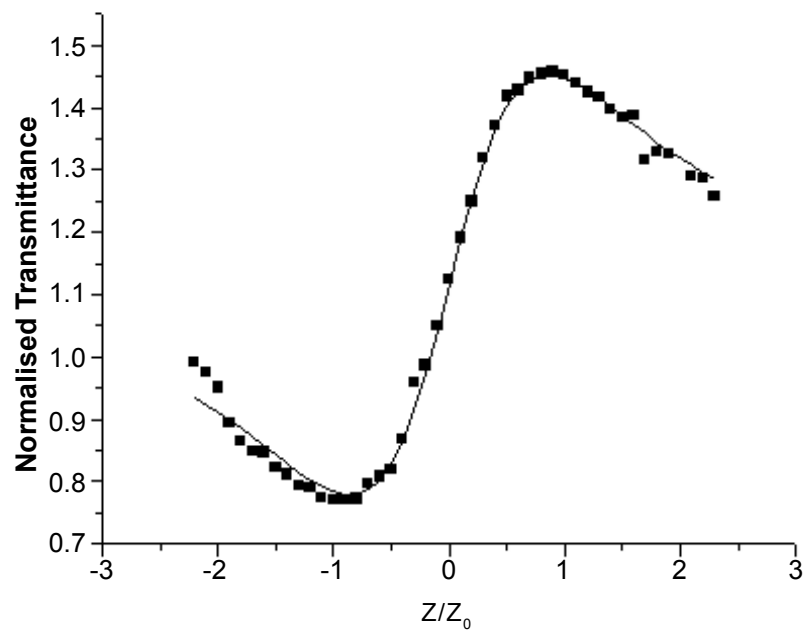

Figure 4: Measured z-scan of the dye Victoria blue in nBA The solid line is the calculated result with $\Delta \varnothing=1.72$.

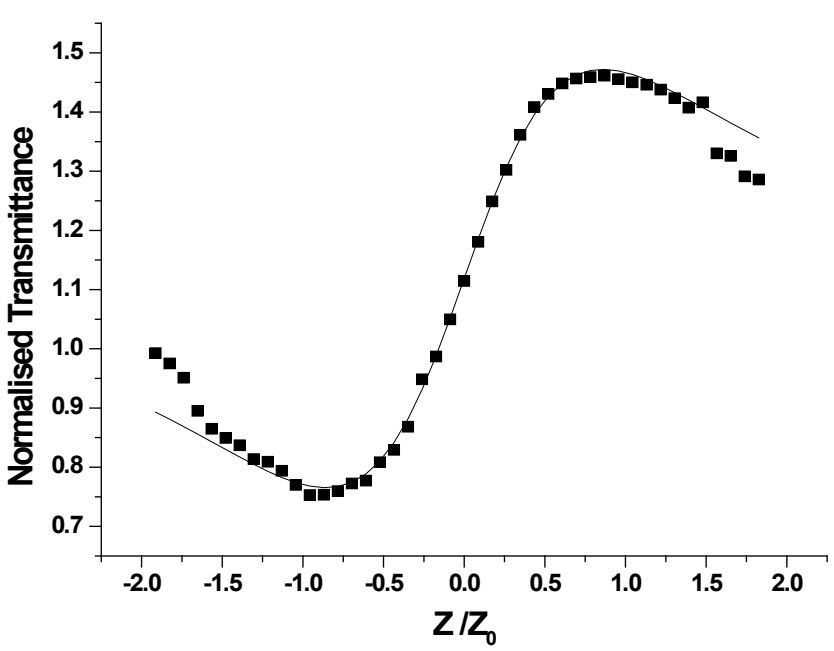

Figure 5: Measured z-scan of the dye Victoria blue doped polymer film. The solid line is the calculated result with $\Delta \varnothing_{0}=1.77$.

ground state and the excited state of the dye molecules. The Stoke's shift also depends on the size, polarity and relaxation time of the additive molecules.

Analysis of nonlinearity in the dye victoria blue shows that the dye has a negative (self-defocusing) nonlinearity. Self-defocusing effect is due to local variation of refractive index with temperature. A pre-focal transmittance minimum (valley) followed by a post-focal transmittance maximum (peak) in the $\mathrm{z}$-scan experiment is a signature of positive nonlinearity. The $\mathrm{z}$-scan signature for the dye victoria blue in $\mathrm{nBA}$ gives the value of the transmission from peak to valley $\left(\Delta \mathrm{T}_{\mathrm{p}-\mathrm{v}}\right)$ is 0.19 , which shows an index change of $\left\langle\Delta \mathrm{n}_{\mathrm{o}}\right\rangle=-5.067 \times 10^{-6}$, calculated using the formula, $\Delta \varnothing_{\mathrm{o}}=\mathrm{k} \Delta \mathrm{n}_{\mathrm{o}} \mathrm{L}_{\text {eff }}$, where $\mathrm{k}$ is the wave vector, $\mathrm{L}_{\text {eff }}\left(1-\mathrm{e}^{-\alpha \mathrm{L}}\right) / \alpha$, with $\mathrm{L}$ the sample length and $\alpha$ the linear absorption coefficient. This value of $\langle\Delta \mathrm{n}\rangle$ corresponds to the value of nonlinear coefficient of refractive index $\gamma=-4.47 \times 10^{-12} \mathrm{~m}^{2} / \mathrm{W}$, found using $\Delta \mathrm{n}_{\mathrm{o}}=\gamma \mathrm{I}_{0}$ with $\mathrm{I}_{0}$ being the onaxis irradiance at focus of the lens. The results are compared with dye doped polymer film which shows the value of transmission from peak to valley $\left(\Delta \mathrm{T}_{\mathrm{p}-\mathrm{v}}\right)$ as 0.201 giving an index change of $\left\langle\Delta \mathrm{n}_{\mathrm{o}}\right\rangle=-5.22 \times 10^{-6}$. This value of $\left\langle\Delta \mathrm{n}_{0}>\right.$ corresponds to the value of $\gamma=-4.61 \times 10^{-12} \mathrm{~m}^{2} / \mathrm{W}$. The optical nonlinearity in this dye may be due to laser heating induced nonlinear effect. A laser beam, while passing through an absorbing media, induces temperature and density gradients that change the refractive index profile. This intensity-induced localized change in the refractive index results in a lensing effect on the optical beam. The value of $\left(\Delta \mathrm{T}_{\mathrm{p}-\mathrm{v}}\right)$ has increased for the dye doped polymer film when compared to the dye in $\mathrm{nBA}$. This may be due to the heat dissipation in liquid is fast when compared to the polymer film. And it is this heat inducing a hike in $\left(\Delta \mathrm{T}_{\mathrm{p}-\mathrm{v}}\right)$ value in polymer film.

\section{Conclusion}

The spectral characteristics of dye victoria blue in liquid and solid environments have been studied in detail. Nonlinear studies show that the Victoria blue show a negative nonlinear refractive index. This dye doped polymer can be utilized in optical limiting, low-power degenerate four wave mixing, data storage and gating applications.

Nonlinear refraction in the membrane is used to obtain optical limiting behaviour in an aperture-limited geometry. The origin of optical nonlinearity observed in the $\mathrm{CW}$ regime is attributed to the thermal variation of local refractive index in the medium.

\section{References}

1. Sheik-Bahae M, Said AA, Tai-Huei W, Hagan J, Van Stryland EW (1990) Sensitive Measurement of Optical Nonlinearities Using a Single Beam. IEEE Journal of Quantum Electronics 26: 760.

2. Somasundaram G, Ramalingam A (2000) Gain studies of Coumarin 1 dyedoped polymer laser. Journal of Lumin 90: 1.

3. Dyanamaev, Manenkov, Maslyukov, Matyushin, Nechitalio et al. (1992) Opt Soc Am B 9: 143.

4. Umakanta T, Rajesh RJ, Prem BB, Subrahamanyam A (2002) Optica nonlinearity of organic dyes as studied by Z-scan and transient grating techniques. Journal of Chemical Sciences 114: 557-564

5. Leite RCC, Porto SPS, Damen TC (1967) The Thermal Lens Effect As A Power-Limiting Device. Appl Phys Lett 10: 100.

6. Justus BL, Huston AL, Campillo AJ (1993) Broadband thermal optical limiter. Appl Phys Lett 63: 1483.

7. Pascale Brochard and Valerie Grolier-Mazza and Regis Caband (1997) J Opt Soc Am B 14: 995

8. Costela, Garcia-marino, Figuera, Amat-Guerri, Barrosoand, Shastre (1996) Opt Comm 130: 44.

9. Govindanunny, Sivaram (1980) J Lumin 21: 397

10. Crosby GA, Demas JN (1971) Measurement of photoluminescence quantum yields. Review. J Phy Chem 75: 991-1024.

11. Kubin RF, Fletcher AN (1982) Fluorescence quantum yields of some rhodamine dyes. J Lumin 27: 455-462. 
Citation: Sindhu SV, Ramalingam A, Srinivasan AG (2015) Measurement of Nonlinearity and Spectral Study of a Laser Dye. J Laser Opt Photonics 2: 121. doi:10.4172/jlop.1000121

Page 4 of 4

12. Amat-Guerri F, Costela A, Figuera JM, Florida F, Sastre R (1993) Laser action from rhodamine $6 G$ doped poly (2-hydroxyethyl methacylate) matrices with different crosslinking degrees. Chem Phy Lett 209: 352-356.
13. Costela A, García-Moreno I, Sastre R, Coutts DW, Webb CE (2001) High repetition rate polymeric solid-state dye lasers pumped by a copper-vapor laser. Appl Phys Lett 79: 452 